\title{
Surface plasmon illumination scheme for contact lithography beyond the diffraction limit
}

\author{
Olivier J.F. Martin* \\ Electromagnetic Fields and Microwave Electronics Laboratory, Swiss Federal Institute of Technology, ETH-Zentrum, \\ ETZ G-96, Gloriastrasse 35, CH-8092 Zurich, Switzerland
}

\begin{abstract}
A novel local illumination scheme for optical lithography is proposed. It is based on the excitation of a surface plasmon on a metal film incorporated into a polymer light coupling mask for contact lithography. The electromagnetic field associated with the surface plasmon generates illumination volumes in the photoresist which are not limited by the diffraction (or Rayleigh) limit. Computer simulations indicate that the replication of $20 \mathrm{~nm}$ features using $630 \mathrm{~nm}$ illumination wavelength can be achieved with this technique.

(C) 2003 Elsevier Science B.V. All rights reserved.
\end{abstract}

Keywords: Surface plasmons; Contact lithography; Contrast mechanism; Scattering calculation

\section{Introduction}

The majority of mask-based optical lithography techniques is limited by the diffraction or Rayleigh limit, which states that light cannot be focused on dimensions much smaller than $d$,

$$
d=k \lambda / \mathrm{NA}
$$

where $\lambda$ is the effective illumination wavelength, NA the numerical aperture of the imaging system, and $k$ a process-dependent parameter, usually not much smaller than unity $[1,2]$.

Within the limits set by the Rayleigh criterion, there are two possible approaches for producing a light spot with a smaller spatial extension: either reduce the wavelength $\lambda$, or increase the numerical aperture NA. In optical lithography, the main trend follows the former approach, using extreme UV or $\mathrm{X}$-rays and electrons as light sources [3]. Note in passing that Eq. (1) also sets the limit for optical data storage, where the achievable information density is determined by the illumination spot. Recent developments in optical data storage tackle the Rayleigh limit from both sides, by simultaneously

*Fax: +41-1-632-1647.

E-mail address: martin@ifh.ee.ethz.ch (O.J.F. Martin). 
reducing the wavelength $\lambda$ using light sources in the blue, and increasing the numerical aperture NA with solid immersion lenses [4].

It is however possible to go beyond the Rayleigh limit by using near-field optics [5-7]. In near-field optics, the electromagnetic field can be confined to features much smaller than the wavelength $[8,9]$, as illustrated in Fig. 1. Fig. 1 shows the electric field intensity distribution in the air (permittivity $\varepsilon_{\mathrm{a}}=1$ ), at varying distances $z$ above a dielectric object (permittivity $\varepsilon_{\mathrm{d}}=2.25$ ) deposited on a surface with the same permittivity. The system is illuminated from underneath, at a large angle of incidence $\theta_{\mathrm{i}}$, so that the illumination field is totally reflected at the dielectric-air interface (Fig. 1(a), so-called attenuated total reflection, ATR [1]). This generates an evanescent field above the surface, which does not propagate into the superior half-plane. The illumination wavelength in vacuum is $\lambda=630 \mathrm{~nm}$. Fig. 1(b) indicates that the electromagnetic field is strongly localized around the surface protrusion and perfectly reproduces the protrusion shape, although it is much smaller (in this case more than ten times) than the illumination wavelength. This however is purely a near-field effect: when the observation distance increases the field magnitude decreases and the localization effect diminishes, Fig. 1(b). At larger observation distances, only a weak scattering signal is recorded.

The physical origin of this localization phenomenon is illustrated in Fig. 1(a). The incident field has been chosen with the so-called parallel polarization, where the incident electric field $E^{0}$ is parallel to the plane of incidence [1]. This dominant field component is normal to the protrusion top surface. The boundary conditions imposed by Maxwell's equations to such a normal electric field require the displacement field $D=\varepsilon E$ to be continuous across the dielectric-air interface [10]. Since the permittivity $\varepsilon_{\mathrm{d}}$ in the material is larger than that $\varepsilon_{\mathrm{a}}$ of air above the object, a strong electric field $E^{\mathrm{d}}$ is created just above the surface. Its magnitude is larger than that of the field under the surface by a factor $\varepsilon_{\mathrm{d}} / \varepsilon_{\mathrm{a}}$. One sometimes refers to this field as depolarization field [11].

From the preceding, we retain that the electromagnetic field can be localized on dimensions much smaller than the wavelength, provided that a material contrast exists and the appropriate polarization is at hand. (This localization effect is not observed for the other polarization, so-called s-polarization, where the incident electric field is normal to the plane of Fig. 1(a), and therefore parallel to the protrusion top surface [9].)

The objective of this paper was to investigate a possible implementation of this near-field illumination scheme using a realistic lithography technique, which allows parallel illumination of

(a)

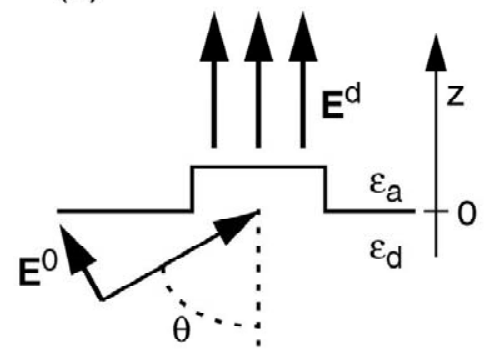

(b)

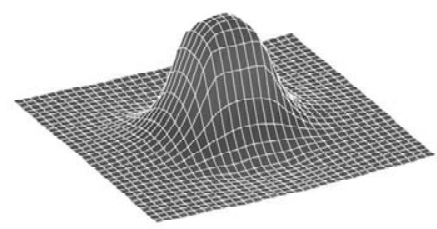

(c)

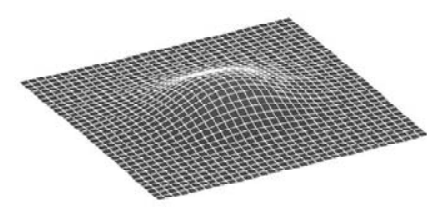

Fig. 1. Light confinement in near-field optics. (a) A $20 \times 20 \times 10 \mathrm{~nm}$ protrusion deposited on a glass substrate is illuminated under attenuated total reflection with $\lambda=630 \mathrm{~nm}$ wavelength. A depolarization field $E^{\mathrm{d}}$ is created above the protrusion. Electric field intensity distribution (b) $5 \mathrm{~nm}$, respectively (c) $15 \mathrm{~nm}$, above the protrusion ( $z=15 \mathrm{~nm}$, respectively $z=25 \mathrm{~nm}$ ). The lateral dimensions of the intensity maps are $80 \times 80 \mathrm{~nm}^{2}$. 
features beyond the diffraction limit. This implementation, based on light coupling masks, is discussed in Section 2. Achievable resolution and contrast are addressed in Section 3 and concluding remarks given in Section 4. All the results presented here have been obtained with the Green's tensor technique, which provides a flexible framework for scattering calculations in stratified media with embedded scatterers $[12,13]$.

\section{Surface plasmon illumination and contact lithography}

Light coupling masks (LCMs) for contact lithography are structured siloxan polymer masks, where the features to be replicated in the photoresist correspond to protrusions on the mask surface, Fig. 2(a). The material properties of the polymer allow the mask to form conformal contact with the photoresist layer, without the surfaces to wear or be damaged. Since contact between mask and resist only occurs at the protrusion surfaces, light is differentially guided through the protrusions when the structure is illuminated from the top, Fig. 2(a). Using $\lambda=248 \mathrm{~nm}$ illumination wavelength, it is possible to write features in the $80 \mathrm{~nm}$ range with this technique [14-16]. A similar approach, using the interference of the field propagating through the protrusion with that propagating through the air, has also been used to expose the resist on narrow lines corresponding to the spatial derivatives of the mask profile [17].

Lithography based on LCMs is intrinsically diffraction limited: the focusing provided by the mask disappears as soon as the protrusion width $w$ becomes smaller than half the effective wavelength $\lambda_{\text {eff }}$ in the mask $\left(\lambda_{\text {eff }}=\lambda /\left(\varepsilon_{\text {mask }}\right)^{1 / 2}\right.$, where $\varepsilon_{\text {mask }}$ is the mask permittivity). In that case, light cannot be guided through the protrusion and scattering becomes dominant.

To use LCMs beyond the diffraction limit, we incorporate a surface plasmon illumination scheme, as illustrated in Fig. 2(b). Surface plasmons are resonant electron oscillations, which occur at a metal-dielectric interface for specific illumination wavelengths [18]. They can be excited at the metal-air surface of a metal film deposited on a substrate and illuminated through the substrate, Fig. 2(b). A surface plasmon can be viewed as an electromagnetic wave traveling parallel to the metal surface, with an electric field that decays in the air, Fig. 2(b). Various metals support surface plasmons: gold, silver and copper in the visible; aluminium in the UV; different compounds, such as

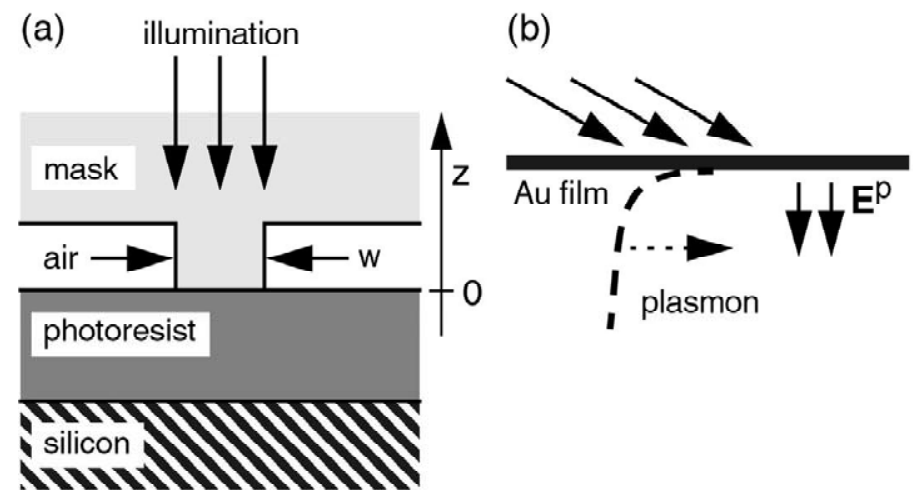

(c)

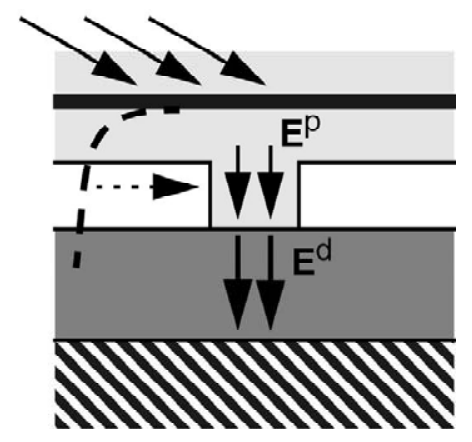

Fig. 2. (a) Light coupling mask (LCM) and its utilization for contact lithography. (b) A surface plasmon can be excited at the lower surface of a metal film deposited on a substrate. (c) LCM incorporating a surface plasmon illumination scheme. 
indium tin oxide, in the infrared. Additional means, like for example a grating deposited on the top metal surface, can be used to enhance the coupling between the illumination field and the surface plasmon.

In our exposure scheme, the plasmon-supporting layer is incorporated in the LCM, Fig. 2(c). When the structure is illuminated from the top, a surface plasmon is generated at the bottom metal surface. The electric field $E^{\mathrm{p}}$ associated with the surface plasmon is vertically polarized, i.e. it is normal to the metal surface. When this field interacts with the protrusion-photoresist interface, a strong depolarization field $E^{\mathrm{d}}$ is created, Fig. 2(c). It is this field that exposes the photoresist. As in the example of Fig. 1(a), the magnitude of the depolarization field in Fig. 2(c) depends on the dielectric contrast between the mask and the photoresist. It is therefore necessary that the permittivities of the LCM and photoresist differ. This represents an important difference from the standard utilization of LCMs, where the index of the mask is matched to that of the photoresist, in order to suppress any back-reflections at their interface [15].

Fig. 3 shows the electric field intensity distribution in an exposure set-up using a LCM under plasmon illumination. The illumination wavelength is $\lambda=630 \mathrm{~nm}$, the mask has permittivity $\varepsilon=2.9$; it incorporates a 20-nm thick gold layer (complex permittivity $\varepsilon=-11.6+i 1.26$ ) deposited on a higher index mask substrate $(\varepsilon=4)$. The photoresist is $200 \mathrm{~nm}$ thick and its permittivity $\varepsilon=2$; it is deposited on a silicon substrate $(\varepsilon=15)$. These permittivity values correspond to the material values at the illumination wavelength $\lambda=630 \mathrm{~nm}$. The complex permittivity used for gold accounts for losses in the metal [19]. The mask protrusion in Fig. 3 has a width $w=60 \mathrm{~nm}$; the air gap between photoresist and recessed parts of the mask has a 60-nm thickness.

The illumination field generates a surface plasmon at the bottom gold surface, which produces an important field in the lower part of the mask (Fig. 3). Due to the index contrast, large depolarization fields are created in the air, at the mask-air interfaces. The evanescent character of the plasmon field prevents these fields from exposing the resist. A depolarization field is also created at the mask-resist interface, under the protrusion. The lateral extension of this field is entirely determined by the width $w$

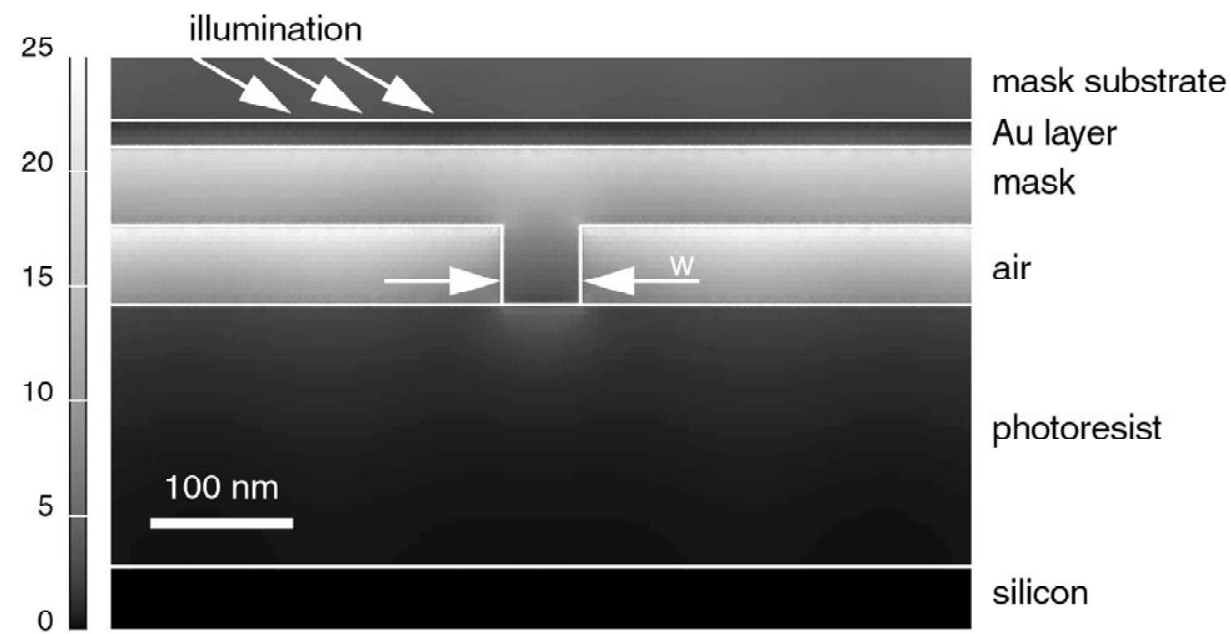

Fig. 3. Electric field intensity distribution in the structure of Fig. 2(c). The surface plasmon illumination generates a localized field in the photoresist, below the protrusion. Illumination wavelength: $\lambda=630 \mathrm{~nm}$, see the text for the dimensions. 
of the protrusion, so that a localized field is created in the resist, irrespective of the illumination wavelength.

\section{Resolution and contrast}

Fig. 4 shows electric field intensity profiles measured in Fig. 3 at different depths $z$ in the photoresist, below the protrusion. A strong light confinement in the photoresist is visible in Fig. 4. In the top of the photoresist, the electric field intensity perfectly reproduces the protrusion, with a full width half-maximum of $65 \mathrm{~nm}$. The field remains well confined deeper in the photoresist, although the contrast rapidly diminishes. This can, however, be overcome by using a very thin photoresist or surface imaging techniques [20-22].

The resolution that can be achieved with this approach is investigated in Fig. 5(a), where the electric field intensity in the photoresist $(z=-10 \mathrm{~nm})$ is shown for different protrusion widths $w$. Remarkably, the field distributions follow the protrusion width $w$, even for protrusions as small as $w=20 \mathrm{~nm}$. Keep in mind that all these results are obtained for an illumination wavelength of $\lambda=630 \mathrm{~nm}$. They demonstrate that it is possible to expose the photoresist on volumes with dimensions much smaller than the diffraction limit. Larger structures can also be replicated, as illustrated in Fig. 5 for $w=80 \mathrm{~nm}$ and $w=100 \mathrm{~nm}$. For protrusions in the $w=20-80 \mathrm{~nm}$ range, the overall field distribution very well reproduces the entire protrusion, with a square distribution showing a flat top. Further, intensity and contrast are very similar for these different protrusions widths. A dip starts however to appear for the largest protrusion $w=100 \mathrm{~nm}$.

One could wonder whether the energy associated with $\lambda=630 \mathrm{~nm}$ illumination wavelength is too small for high resolution photoresist. Advanced chemical approaches, such as two photons processes [23], should make possible the fabrication of efficient photoresists for this wavelength. However, from a physical point of view, the proposed plasmon illumination scheme can also function at a shorter wavelength, as illustrated in Fig. 5(b). This figure shows the electric field intensity in the photoresist

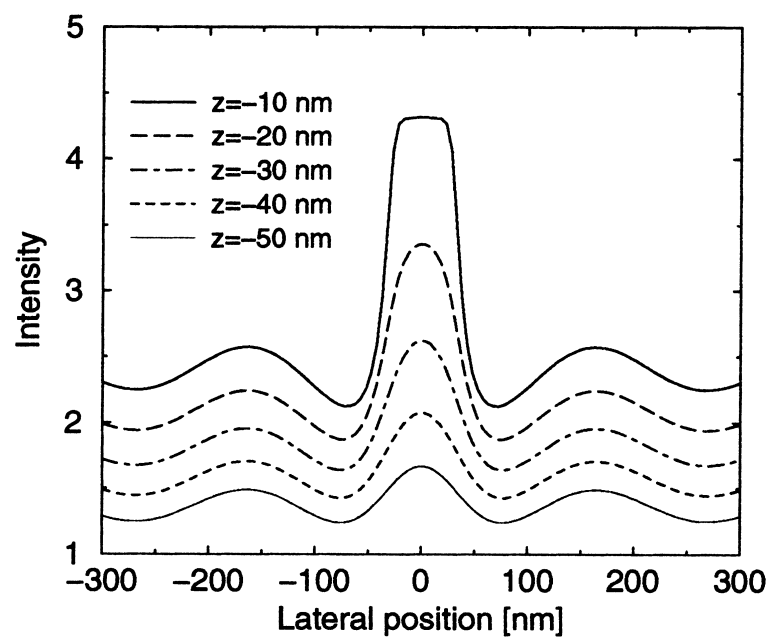

Fig. 4. Electric field intensity at different depths $z$ in the photoresist, below the protrusion (see Fig. 3). 

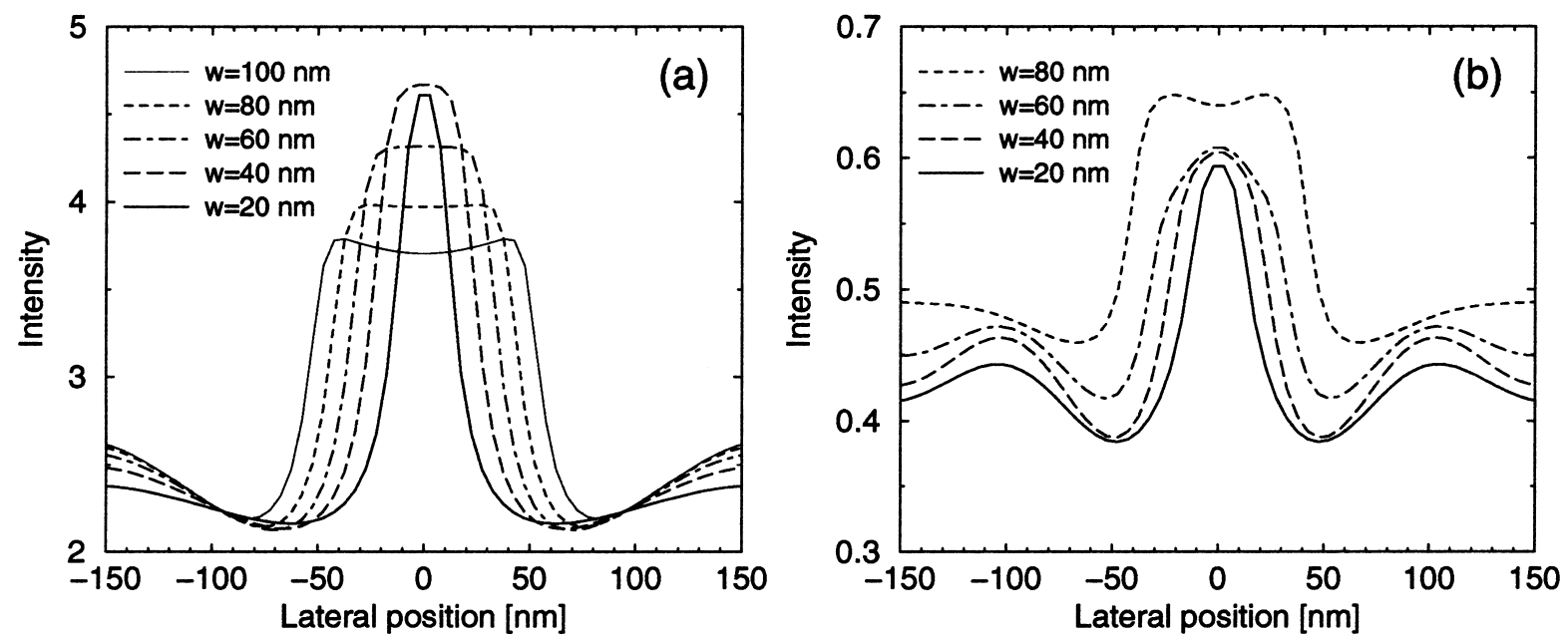

Fig. 5. Electric field intensity in the photoresist $(z=-10 \mathrm{~nm}$ ), below the protrusion, for different protrusion widths $w$ (see Fig. 3). (a) $\lambda=630 \mathrm{~nm}$ illumination and (b) $\lambda=248 \mathrm{~nm}$ illumination wavelength.

$(z=-10 \mathrm{~nm}$ ), for different protrusion widths $w$ and an illumination wavelength of $\lambda=248 \mathrm{~nm}$. The mask parameters were taken the same as in the previous figure, except for the permittivity of gold $(\varepsilon=0.87+i 4.3[19])$. Again the profiles in the photoresist perfectly reproduce the protrusions for $w=20-60 \mathrm{~nm}$. For wider protrusions, where $w$ becomes comparable to half the effective wavelength in the photoresist (approximatively $85 \mathrm{~nm}$ ), one quits the evanescent regime and the replication becomes less good.

\section{Conclusion}

The calculations presented in this paper indicate that it is possible to develop a lithography technique which is not bound to the diffraction limit and allows the replication of extremely small features using visible light illumination. The proposed implementation, which combines plasmon illumination with a mask technique readily available, should be feasible with today's technology. In addition to creating an electromagnetic field with the required polarization, the proposed plasmon illumination scheme allows decoupling the broad global illumination of the entire mask from the localized exposure of the photoresist at specific locations. Like this, scattering and background noise can be suppressed.

\section{Acknowledgements}

It is a pleasure to acknowledge stimulating discussions with M. Paulus. This work was supported by the Swiss National Science Foundation. 


\section{References}

[1] M. Born, E. Wolf, Principles of Optics, 6th Edition, Pergamon Press, Oxford, 1987.

[2] Special Issue on Optical Lithography, IBM J. Res. Dev. 41(1/2) (1997).

[3] I.R. Committee and I.T.W. Groups, Technical report, Semiconductor Industry Association (2001) http://public.itrs.net.

[4] S. Imanish, T. Ishimoto, Y. Aki, T. Kondo, K. Kishima, K. Yamamoto, M. Yamamoto, Jpn. J. Appl. Phys. 39 (2000) 800.

[5] D.W. Pohl, W. Denk, M. Lanz, Appl. Phys. Lett. 44 (1984) 651.

[6] E. Betzig, J.K. Trautman, T.D. Harris, J.S. Weiner, R.L. Kostelak, Science 251 (1991) 1468.

[7] J.-C. Weeber, E. Bourillot, A. Dereux, J.-P. Goudonnet, Y. Chen, C. Girard, Phys. Rev. Lett. 77 (1996) 5332.

[8] O.J.F. Martin, C. Girard, A. Dereux, Phys. Rev. Lett. 74 (1995) 526.

[9] O.J.F. Martin, C. Girard, A. Dereux, J. Opt. Soc. Am. A 13 (1996) 1801.

[10] J.D. Jackson, Classical Electrodynamics, 3rd Edition, Wiley, New York, 1999.

[11] J. van Kranendonk, J.E. Sipe, in: E. Wolf (Ed.), Progress in Optics 15, Elsevier, New York, 1977, p. 247.

[12] M. Paulus, O.J.F. Martin, J. Opt. Soc. Am. A 18 (2001) 854.

[13] M. Paulus, O.J.F. Martin, Phys. Rev. E 63 (2001) 066615.1.

[14] J.A. Rodgers, K.E. Paul, R.J. Jackman, G.M. Whitesides, Appl. Phys. Lett. 70 (1997) 2658.

[15] H. Schmid, H. Biebuyck, B. Michel, O.J.F. Martin, Appl. Phys. Lett. 72 (1998) 2379.

[16] H. Schmid, H. Biebuyck, B. Michel, O.J.F. Martin, N.B. Piller, J. Vac. Sci. Technol. B 16 (1998) 3422.

[17] J.A. Rodgers, K.E. Paul, R.J. Jackman, G.M. Whitesides, J. Vac. Sci. Technol. B 16 (1998) 59.

[18] K. Welford, in: Surface Plasmon-Polaritons, No. 9 in IOP Short Meetings Series (IOP, Bristol, 1988) 25.

[19] P.B. Johnson, R.W. Christy, Phys. Rev. B 6 (1972) 4370.

[20] G.N. Taylor, L.E. Stillwagon, T. Venkatesan, J. Electrochem. Soc. 131 (1984) 1658.

[21] J. Aizenberg, J.A. Rodgers, K.E. Paul, G.M. Whitesides, Appl. Phys. Lett. 71 (1997) 3773.

[22] V. Rao, J. Hutchinson, S. Holl, J. Langston, C. Henderson, D.R. Wheeler, G. Cardinale, D. O'Connell, J. Goldsmith, J. Bohland, G. Taylor, R. Sinta, J. Vac. Sci. Technol. B 16 (1998) 3722.

[23] B.G. Sfez, G. Rosenblum, Z. Kotler, V. Lyubin, M. Klebanov, Mat. Sci. Semicond. Process. 3 (2000) 499. 